\title{
Improving beam spectral and spatial quality by double-foil target in laser ion acceleration
}

\author{
C.-K. Huang, ${ }^{*}$ B. J. Albright, L. Yin, H.-C. Wu, K. J. Bowers, B. M. Hegelich, and J. C. Fernández \\ Los Alamos National Laboratory, Los Alamos, New Mexico 87545, USA
}

(Received 16 April 2010; published 8 March 2011)

\begin{abstract}
Mid- $Z$ ion driven fast ignition inertial fusion requires ion beams of hundreds of MeV energy and $<10 \%$ energy spread. The break-out afterburner (BOA) is one mechanism proposed to generate such beams; however, the late stages of the BOA tend to produce too large of an energy spread. Here we show how use of a second target foil placed behind a nm-scale foil can substantially reduce the temperature of the comoving electrons and improve the ion beam energy spread, leading to ion beams of energy hundreds of $\mathrm{MeV}$ and $6 \%$ energy spread.
\end{abstract}

DOI: 10.1103/PhysRevSTAB.14.031301

PACS numbers: 52.38.Kd, 52.57.Kk, 52.65.Rr

Over the past decades, laser acceleration of ions has seen dramatic progress, starting from the demonstration of multi-MeV proton beams from the interaction of high intensity $\left(I \geqq 10^{18} \mathrm{~W} / \mathrm{cm}^{2}\right)$ lasers with targets tens of microns or more in thickness [1]. The dominant ion acceleration in these targets results from "Target Normal Sheath Acceleration" (TNSA) [2], which involves the creation of an electrostatic sheath on the rear target surface from the expulsion of laser-heated electrons from the front of the target. Electric fields in the sheath ionize and accelerate ions at the rear of the target to form multi-MeV ion beams with advantageous beam divergence, emittance, and ionic species properties [3]. Quasimonoenergetic TNSAgenerated ion beams have also been demonstrated [4], but at modest energy.

Several applications require highly energetic, quasimonoenergetic beams. In particular, one approach to ionbased fast ignition [5] employs carbon ion beams [6] with a mean energy $E \sim 450 \mathrm{MeV}$ and an energy spread $\Delta E / E=10 \%$. A promising technique for creating such beams is the break-out afterburner (BOA) [7], which involves the interaction of an ultrahigh contrast laser with a target of nm-scale thickness. Such targets become relativistically transparent, as in simulations [7] and later observed in recent Trident experiments [8], and in the process achieve substantially higher peak ion energy over TNSA with comparable laser intensity and energy. Indeed, the required $\sim 450 \mathrm{MeV}$ carbon peak energies have been experimentally demonstrated with a laser pulse energy as little as $80 \mathrm{~J}$, several orders of magnitude smaller than the tens of $\mathrm{kJ}$ required for fast ignition. In the BOA, the ion beam has a small $\Delta E / E$ early in the evolution; however at late time, $\Delta E / E$ grows and the beam relaxes to a

\footnotetext{
*huangck@lanl.gov

Published by American Physical Society under the terms of the Creative Commons Attribution 3.0 License. Further distribution of this work must maintain attribution to the author(s) and the published article's title, journal citation, and DOI.
}

Boltzmann-like spectrum [7]. If this relaxation can be arrested, BOA-generated ion beams may be used in a wider range of applications.

In the BOA, an intense laser impinges upon an overdense target thin enough that it becomes relativistically transparent during the interaction. This happens when the density of the expanding, rapidly heated foil drops below the relativistic transparency threshold $n_{e} \approx n_{\mathrm{cr}} \gamma$, where $n_{e}$ and $\gamma$ are the density and Lorentz factor for the electrons in the target interacting with the laser and $n_{\mathrm{cr}}=m c \omega_{0}^{2} / 4 \pi e^{2}$ is the critical density for the laser of frequency $\omega_{0}$. Nearly all electrons are in the laser field in this stage; they heat volumetrically and gain substantial forward momentum. During this "break-out afterburner" stage, the ion energy is boosted dramatically. In the early part of BOA, the ions have a quasimonoenergetic energy spectrum, as observed from the 1D particle-in-cell (PIC) simulation result using the VPIC code [9] in Fig. 1.

In this simulation, a linearly polarized laser beam with intensity $I=10^{21} \mathrm{~W} / \mathrm{cm}^{2}$ and wavelength $\lambda=1.0 \mu \mathrm{m}$ encounters a carbon foil of thickness $d=50 \mathrm{~nm}$. We define $t=0$ when the laser is incident on the target. The laser electric field has a rising edge $E_{y}=$ $E_{0} \sin [\pi(t / 106 \mathrm{fs})]$ for $t<53 \mathrm{fs}$ followed by a flattop profile with initial $a_{0}=e E_{0} / m c \omega_{0}=27$. The carbon foil consists of $\mathrm{C}^{6+}$ ions and electrons. The initial electron density is $n_{e 0}=660 n_{\mathrm{cr}}$, and the initial temperature is $5 \mathrm{keV}$. The simulation resolution, time step, and number of particles per cell are $0.02 c / \omega_{p e}, 0.0196 / \omega_{p e}$, and 1000, respectively, which convergence studies show to accurately model the break-out stage. The target is initially placed at $x=5 \mu \mathrm{m}=806 c / \omega_{p e}$. At $t=120 \mathrm{fs}$, the target becomes transparent and an accelerating electric field peaks near the center of the ion beam [Fig. 1(a)]. The $\mathrm{C}^{6+}$ ion energy spectrum has a peak at $196 \mathrm{MeV}$ and an energy spread of $10.7 \%$ at this time, as seen in Fig. 1(b). However, this feature does not persist. The ions near the maximum ion density experience the largest electric field and have the largest energy gain, whereas ions just ahead or behind this density peak gain less energy. This effect leads to a 

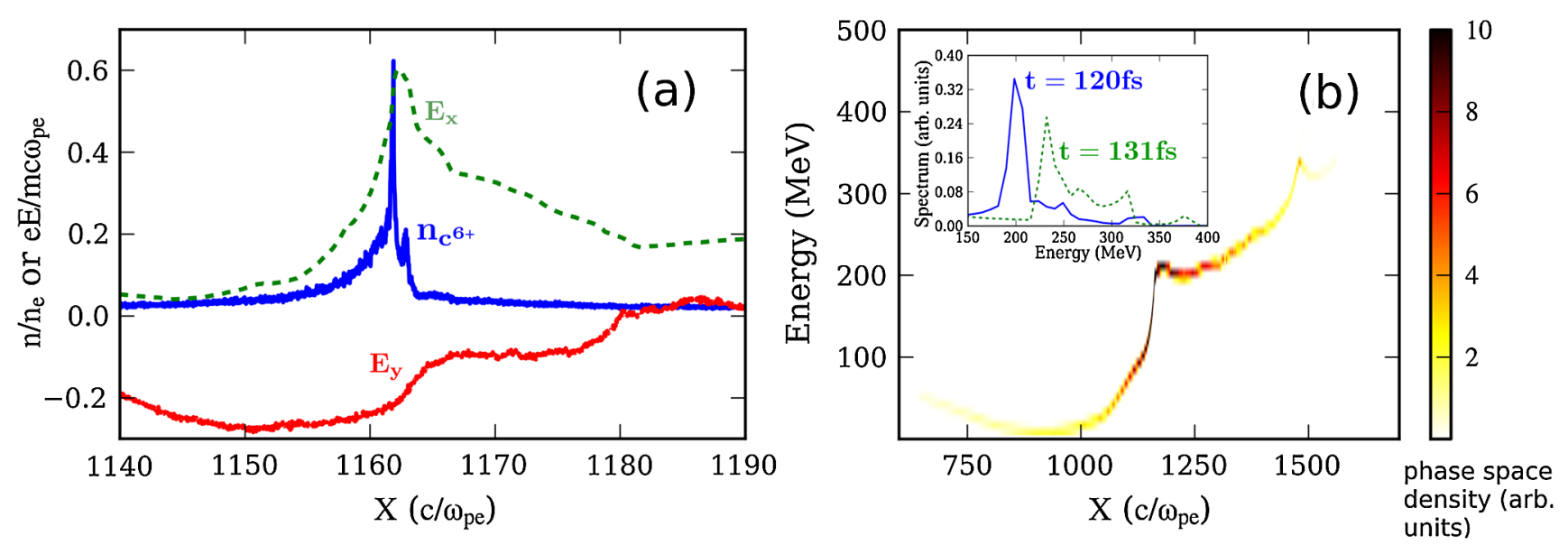

FIG. 1. 1D PIC simulation of the laser thin-foil interaction at the BOA stage. (a) The laser field (polarize in $y$ and propagate from left to right in $x$ ) has penetrated the target whose peak is at $x=1161 c / \omega_{p e}$ at $t=120 \mathrm{fs}$. (b) Phase space of the $\mathrm{C}^{6+}$ ion at $t=120 \mathrm{fs}$ when BOA happens. The energy spectrum (inset) shows a quasimonoenergetic peak at $196 \mathrm{MeV}$ and the FWHM is $21 \mathrm{MeV}$, corresponding to an energy spread of $10.7 \%$. The energy spread evolves to $>30 \%$ at $t=131$ fs and the phase space develops a kink structure (not shown) as the result of the acceleration field $E_{x}$ shown in (a).

kink structure at the beam center in the phase space (opposite of TNSA-generated ion beams, which generally increase monotonically in energy), which causes the energy spread to increase over time as shown in Fig. 1(b). Furthermore, the laser pulse will continue to heat the electrons as it comoves with the target. These hot electrons cause significant expansion of the ion beam.

In this paper, we concentrate on ion dynamics after the BOA stage and show that by adding a second foil with properly chosen thickness, density, and separation distance from the first foil, both the energy spread and the spatial extent of the ion beam can be improved, a vital step toward controllable laser-driven sources. The dynamics of the interactions leading to this controllability is understood through analysis of PIC simulations. (The use of multilayer or spallation targets in the target transparent regime has been considered in [10], though the dynamics is different from that discussed here.)

The second foil serves three purposes: (1) preventing further laser heating of the comoving electrons; (2) acting as a medium to exchange hot electrons for cold ones, and (3) reducing the ion beam's energy spread, i.e., reversing the chirp of the ion beam. First, the overdense foil prevents the laser from interacting with the electrons comoving with the ions, thereby preventing further heating of the electrons surrounding the ion beam while allowing the ions to penetrate. This is illustrated in Fig. 2(a) schematically and in 2(b) using a 1D VPIC simulation with the same simulation parameters as in Fig. 1, but with a second foil. We now use subscript $1 / 2$ to denote the first/second foil, 0 to denote initial quantities, and $e / i$ to denote electron/ion in the targets. The second foil is at $x_{2}-x_{1}=4 \mu \mathrm{m}$ from the first foil. (In our analysis, we also assume that the second foil becomes fully ionized under combined effects from the irradiation of the intense laser pulse, the field ionization
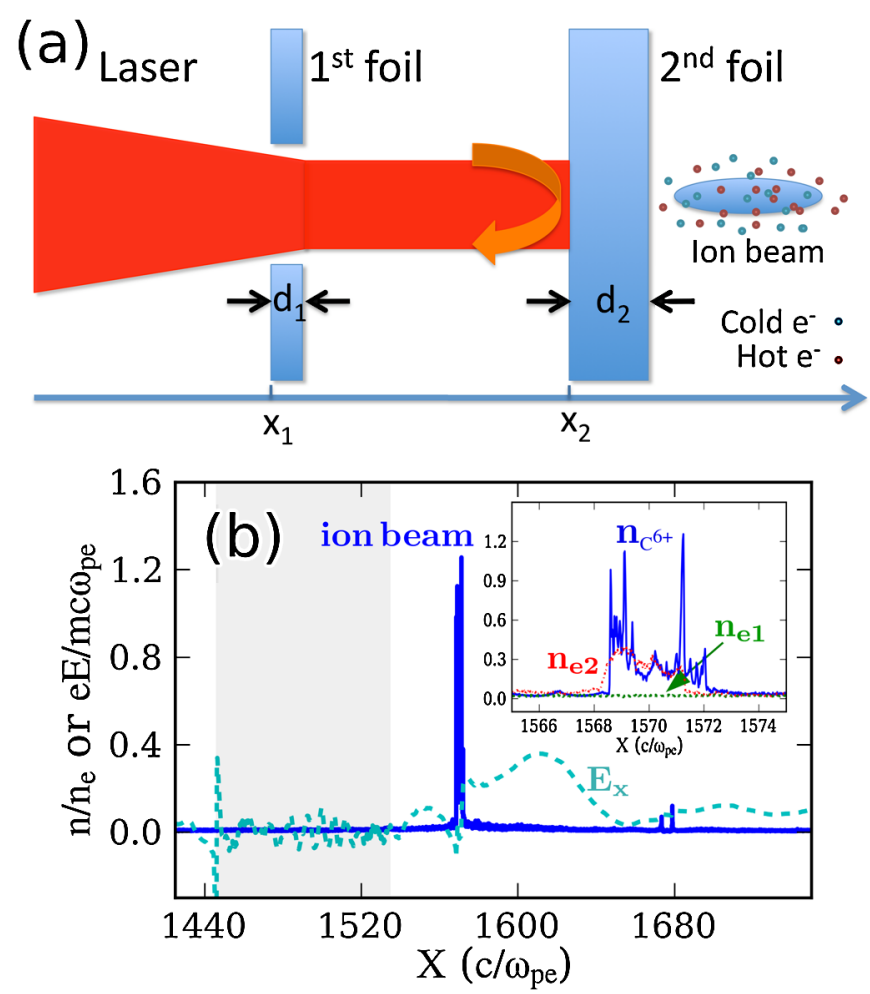

FIG. 2. (a) Diagram of the double-foil configuration. The laser is reflected at the second foil while the ion beam comoves with electrons of two-temperature components. (b) 1D PIC simulation with the second foil (shaded region) of thickness $d_{2}=0.5 \mu \mathrm{m}$ and placed at $x_{2}=9 \mu \mathrm{m}=1452 c / \omega_{p e}$ (laser field/first foil not shown). The TNSA sheath field (dashed, light blue) is detached from the second foil and comoves with the ions (solid, blue) which is spatially localized at $x=1570 c / \omega_{p e}$ with a width $d^{\prime} \sim 4 c / \omega_{p e}<d_{1}$ (see inset) at $t=156 \mathrm{fs}$. The inset also shows the comoving electrons from the first foil (dashed, green)/second foil (dotted, red). 
from the charge separation field, and the electron impact ionization $[10,11]$. This is a reasonable approximation for laser intensity $\sim 10^{21} \mathrm{~W} / \mathrm{cm}^{2}$, provided the foil is composed of low- $Z$ to mid- $Z$ material.) The second foil has sufficient density and thickness such that it neither turns transparent nor incurs large longitudinal motion under the irradiation of the part of the laser pulse that penetrates the first foil. The former condition implies that $n_{e 2} \gg n_{\mathrm{cr}}$ and $d_{2} \gg c / \omega_{p e}$, where $n_{e 2}$ and $d_{2}$ are the density and thickness of the second foil, respectively. The ion beam and the copropagating electrons have charge density lower than the critical density due to target expansion (but are still approximately charge-neutral macroscopically) so they do not excite a large perturbation in the second foil. Therefore the longitudinal electric field inside the second foil is small. However, the second foil should not be so thick that it stops the ion beam through collisional or collisionless processes. In practice, we find a carbon foil of thickness $\sim 1 \mu \mathrm{m}$ satisfies these considerations. Here we use a second carbon foil of $d_{2}=0.5 \mu \mathrm{m}$ and of electron density $n_{e 2}=660 n_{\mathrm{cr}}$. The laser stops in the target within a few skin depths. Figure 2(b) shows that the ion beam emerges at the right side of the foil at $x=1570 c / \omega_{p e}$ when $t=$ $156 \mathrm{fs}$. As the ion beam does not experience any significant collective acceleration field inside the second foil, the ions free-stream through the foil. Electrons from the first foil with sufficient forward momentum also penetrate the second foil and comove with the ion beam [Fig. 2(b)].

Upon entering the second foil, the ion beam has a characteristic energy phase space where the front part of the ion beam has less energy and moves at a slower velocity $v_{\text {front }}$ and the middle part of the ion beam has more energy and moves at a faster velocity $v_{\text {mid }}$. This energy chirp compresses the beam longitudinally during propagation. The beam's longitudinal dimension become smallest after a time interval $t_{c}=d^{\prime} /\left(v_{\text {mid }}-v_{\text {front }}\right)$, where $d^{\prime}$ is the characteristic width of the expanded target. This effect, as shown in Fig. 2(b), has impact on the optimal position and thickness of the second foil for reducing the ion beam's energy spread as we discuss later.

The second function is to exchange hot electrons from the first foil with colder electrons at the back surface of the second foil. In the Fig. 2(b) inset, two groups of electrons comoving with the ions (from the first/second foil, respectively) are plotted. These two groups of electrons emerge from the second foil and propagate with the ion beams, but they possess different characteristics. The first foil electrons, as indicated by the dashed (green) curve in Fig. 2(b), are heated directly by the laser during the break-out stage and have high temperature. In the Fig. 3 inset, the energy distribution of these electrons at the time when the ion beam and comoving electrons arrive at the front surface ( $t=142 \mathrm{fs}$ ) is shown. For reference, the energy distribution is calculated for first foil electrons in a slice three skin depths wide at the front side of the second foil [12].

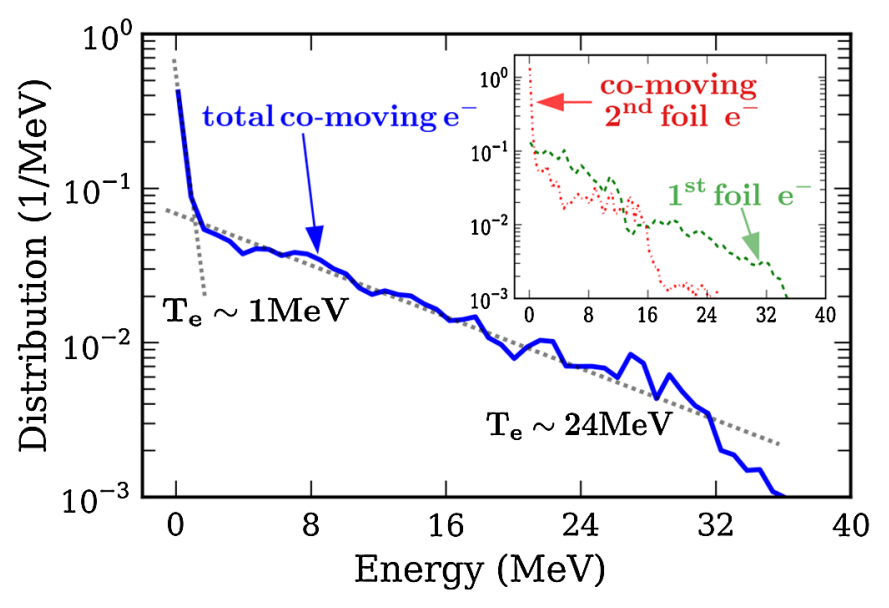

FIG. 3. The energy distribution of the comoving electrons (solid, blue), the electrons from the first foil (dashed, green), and the second foil (dotted, red). The distribution of the electrons from the first foil is measured at the front side of second foil for a slab with thickness of three skin depths at $t=142 \mathrm{fs}$. The distributions of the second foil electrons and the comoving electrons are taken around the ion beam between $x=$ $1550 c / \omega_{p e}$ and $x=1650 c / \omega_{p e}$ (see Fig. 2) at $t=156 \mathrm{fs}$.

Electrons in this slice have a temperature $T_{e} \sim 24 \mathrm{MeV}$ and $E_{k, \max } \simeq 36 \mathrm{MeV}$, consistent with the electron temperature in the laser field.

As in TNSA, fast electrons traverse the second foil and form an electron sheath layer at the back surface. The ions' velocities are smaller than those of the fast electrons, so they lag behind and cannot neutralize the electron sheath immediately. A positive longitudinal electric field at the back surface is generated. This sheath field $E \sim m c \omega_{p} / e$ has a width of several skin depths and acts as a potential barrier for electrons with energy at or below a few $\mathrm{MeV}$. Fast electrons can overcome this barrier and eventually exit the second foil; however, slower electrons are trapped within the second foil.

When the ion beam emerges from the second foil, its positive charge will lower the potential barrier and drag electrons away from the second foil. These electrons and the fast electrons from the second foil that escape the potential barrier form the second group of electrons which comoves with the ion beam [Fig. 2 inset]. The kinetic energy distribution of this group of electrons is shown as the dotted (red) curve in Fig. 3. The distribution has two temperatures, with the bulk having temperature $1 \mathrm{MeV}$ and a halo with temperature $20 \mathrm{MeV}$. The $E_{k \text {, max }} \sim 24 \mathrm{MeV}$ is consistent with the energy of the electrons which are heated by the laser at the front surface of the second foil and escaping the potential barrier in the sheath. However, the emission of hot electrons during the ion beam passage is insignificant compared with the amount of hot electrons from the first foil and cold electrons from the second foil (the lateral emission of TNSA electrons/ions can be used as an experimental signature for this process and may aid in 
determining the optimal laser pulse duration). The total energy distribution of the comoving electrons is plotted as the solid (blue) curve in Fig. 3. It also exhibits a characteristic two-temperature feature, i.e., colder electrons with $T_{e} \sim 1 \mathrm{MeV}$ dominate the spectrum below $2 \mathrm{MeV}$ and hotter electrons with $T_{e} \sim 24 \mathrm{MeV}$ above $2 \mathrm{MeV}$. Comparing this energy distribution with the one for the incident electrons from the first foil (dashed, green curve) in Fig. 3, one can conclude that a significant fraction of the comoving hot electrons have been replaced with cold electrons from the second foil. The presence of a cold electron population would lead to slower expansion of the ion beam. For a two-temperature population of electrons, the ion sound speed, which sets the expansion time scale, has the form $c_{s}=\left[Z_{i}\left(n_{e c}+n_{e h}\right) /\left(n_{e c} / k T_{e c}+\right.\right.$ $\left.\left.n_{e h} / k T_{e h}\right) m_{i}\right]^{1 / 2}$ [13], where subscripts $c / h$ denote cold/ hot components. In the simulation of Fig. $3, c_{s}=0.024 c$, while $c_{s}=0.11 c$ without the second foil. The slower expansion will retain a quasimonoenergetic spectrum for longer, which is preferable for applications such as ion fast ignition.

The third function of the second foil is to control the longitudinal phase space of the ion beam. This is accomplished through the TNSA field at the back of the second foil. We note that unlike the classical TNSA, the comoving first foil electrons (over)compensate charge imbalance at the front side of the second foil created by the laser, thus making an accelerating field at the front side too. The front of the ion beam is accelerated in the TNSA field, which, as noted earlier, reverses the chirp of the BOA ion beam. When the ion beam leaves the second foil, the TNSA sheath detaches from the back surface as shown in [3] and the sheath electric field moves with the slower, front portion of the ion beam, while the faster, rear portion of the ion beam sees a smaller (or even decelerating) field [Fig. 2(b)]. This field pattern reduces the energy difference between the front and rear of the beam incurred during the

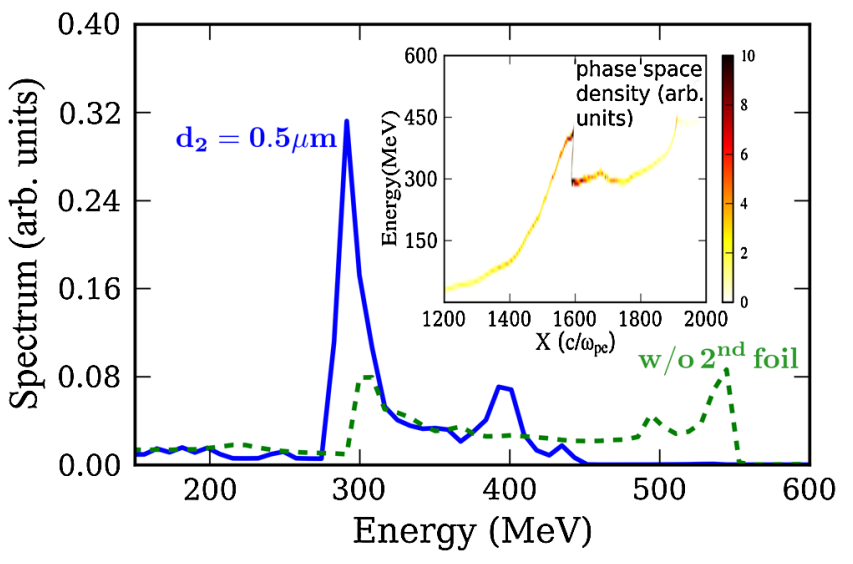

FIG. 4. Energy spectrum of the ion beam with (solid blue) and without (dashed, green) the second foil at $t=158 \mathrm{fs}$. The spectrum shows a quasimonoenergetic peak with $\Delta E / E=6.4 \%$ at $290 \mathrm{MeV}$ for the second foil thickness $d_{2}=0.5 \mu \mathrm{m}$. The inset shows the phase space of the ion beam at $t=158 \mathrm{fs}$.

BOA and leads to smaller $\Delta E / E$. In our simulations, $\Delta E / E$ can be made $<10 \%$ as shown in Fig. 4 for an ion beam with mean energy of hundreds of $\mathrm{MeV}$.

This modification of the ion phase space must occur before fast ions catch up with slower ions, i.e., before the ion beam becomes localized in space. This fundamentally limits the maximum thickness and location of the second foil to be $\sim \mu \mathrm{m}$ for an $I \simeq 10^{21} \mathrm{~W} / \mathrm{cm}^{2}$ laser pulse. On the other hand, as seen in PIC simulations, if the foil thickness is too small (smaller than the expanded target width), the ion beam does not experience significant spatial compaction and the reacceleration in the TNSA field is less effective. These two bounds indicate an optimal thickness of the second foil to achieve the smallest energy spread. Figure 4 shows that $d_{2}=0.5 \mu \mathrm{m}$ is close to this optimal thickness, producing an ion spectrum with a central energy $E_{c} \equiv\left(E_{\max , 1 / 2}+E_{\min , 1 / 2}\right) / 2=290 \mathrm{MeV}$ and an energy
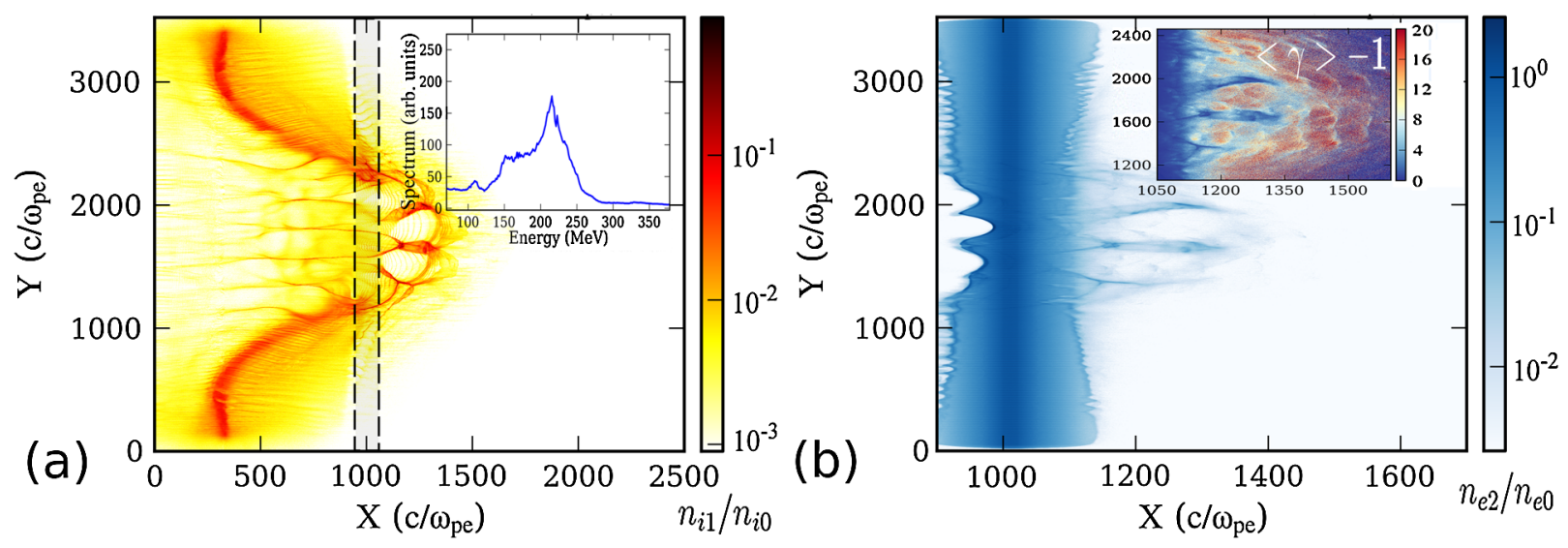

FIG. 5. (a) The first foil ion density $\left(n_{i 1} / n_{i 0}\right)$ at $t=175 \mathrm{fs}$. The dashed lines indicate the location of the second foil. The inset is the energy distribution of the ion beam between $1460 c / \omega_{p}<y<2060 c / \omega_{p}$, the ion beam has $E_{c}=207 \mathrm{MeV}$ and $\Delta E / E=31 \%$. (b) The second foil electron density $\left(n_{e 2} / n_{e 0}\right)$ showing comoving electrons. The inset is the average kinetic energy $(\langle\gamma\rangle-1)$ of the second foil electrons, with cold electrons in blue and hot electrons in red. 
spread $\Delta E / E \equiv\left(E_{\max , 1 / 2}-E_{\min , 1 / 2}\right) / E_{c}=6.4 \%$, where $E_{\min , 1 / 2}$ and $E_{\max , 1 / 2}$ are the low and high energies at half the value of the highest spectrum peak. For nonideal cases of $d_{2}=0 \mu \mathrm{m}, 0.1 \mu \mathrm{m}$, and $1.0 \mu \mathrm{m}(0 \mu \mathrm{m}$ denotes no second foil), simulations show $E_{c}=422 \mathrm{MeV}, 329 \mathrm{MeV}$, and $326 \mathrm{MeV}, \Delta E / E=60 \%, 29 \%$, and $61 \%$, respectively; therefore, one can exchange mean energy for energy spread to some degree with a second foil.

To verify the functions of the second foil in multiple dimensions, we conduct 2D PIC simulations with different laser intensities $\left(1 \times 10^{21} \sim 1.4 \times 10^{21} \mathrm{~W} / \mathrm{cm}^{2}\right)$, target temperatures $(5 \sim 40 \mathrm{keV})$, and numerical resolutions. All simulation results indicate that the functions of the second foil are operative in 2D. The 2D simulation with the same laser and plasma parameters as in 1D simulations (except for a Gaussian laser intensity spot size of $5 \mu \mathrm{m}$ in $y$ and a plasma electron temperature of $40 \mathrm{keV}$ ) is shown in Fig. 5. The laser is polarized along $z$ and is focused at the front side of the first foil, which is placed at $x_{1}=2 \mu \mathrm{m}$. The simulation box size is $11 \mu \mathrm{m}(x) \times 21.9 \mu \mathrm{m}(y)$ and is divided into $21154 \times 42000$ cells with a resolution of $0.084 c / \omega_{p e} \times 0.084 c / \omega_{p e} ; 50$ particles/cell is used for each species. An ion beam of $E_{c}=207 \mathrm{MeV}$ and $\Delta E / E=31 \%$ is observed at $t=175$ fs.

As a final point, we note that our technique can in principle be applied to other ion acceleration techniques, such as radiation pressure acceleration [14], where target bending and associated electron heating are problematic. These effects may be controlled by use of a second foil.

\section{ACKNOWLEDGMENTS}

We acknowledge useful discussions with T. J. T. Kwan and M.H. Key. This work was performed under the auspices of DOE by the Los Alamos National Security/Los Alamos National Laboratory (LANL), and supported by
LDRD. Simulations were performed on Institutional Computing facilities/Roadrunner at LANL and Jaguar at Oak Ridge National Laboratory.

[1] A. P. Fews et al., Phys. Rev. Lett. 73, 1801 (1994); E. Clark et al., ibid. 85, 1654 (2000); R. Snavely et al., ibid. 85, 2945 (2000); A. Maksimchuk et al., ibid. 84, 4108 (2000).

[2] S. P. Hatchett et al., Phys. Plasmas 7, 2076 (2000).

[3] E. Brambrink et al., Phys. Rev. Lett. 96, 154801 (2006); T. Cowan et al., ibid. 92, 204801 (2004); B. M. Hegelich et al., ibid. 89, 085002 (2002); M. Schollmeier et al., ibid. 101, 055004 (2008); J. Schreiber et al., Appl. Phys. B 79, 1041 (2004).

[4] B. M. Hegelich et al., Nature (London) 439, 441 (2006).

[5] M. Roth et al., Phys. Rev. Lett. 86, 436 (2001); M. Temporal et al., Phys. Plasmas 9, 3098 (2002).

[6] J. C. Fernández et al., Nucl. Fusion 49, 065004 (2009).

[7] L. Yin et al., Laser Part. Beams 24, 291 (2006); Phys. Plasmas 14, 056706 (2007).

[8] A. Henig et al., Phys. Rev. Lett. 103, 045002 (2009).

[9] K. J. Bowers et al., Phys. Plasmas 15, 055703 (2008).

[10] A. Gonoskov et al., Phys. Rev. Lett. 102, 184801 (2009); A. Zhidkov et al., Appl. Phys. A 73, 741 (2001).

[11] A. Zhidkov and A. Sasaki, Phys. Plasmas 7, 1341 (2000); G. M. Petrov et al., Plasma Phys. Controlled Fusion 51, 095005 (2009).

[12] The reference position is chosen inside the second target to avoid complication from interaction of the laser field or the sheath field behind the second target.

[13] L. M. Wickens and J.E. Allen, J. Plasma Phys. 22, 167 (1979).

[14] T. Esirkepov et al., Phys. Rev. Lett. 92, 175003 (2004); X. Yan et al., ibid. 100, 135003 (2008); X. Zhang et al., Phys. Plasmas 14, 073101 (2007); O. Klimo et al., Phys. Rev. ST Accel. Beams 11, 031301 (2008); A. P. L. Robinson et al., New J. Phys. 10, 013021 (2008). 